\title{
No-Reference Retinal Image Sharpness Metric Using Daubechies Wavelet Transform
}

\author{
P. Vonghirandecha ${ }^{1}$, P. Bhurayanontachai ${ }^{2}$, S. Kansomkeat ${ }^{1}$, S. Intajag ${ }^{1}$ \\ ${ }^{1}$ Division of Computational Science, Faculty of Science, Prince of Songkla University, Songkhla, \\ Thailand. (e-mail: preecha.v@psu.ac.th) \\ ${ }^{2}$ Department of Ophthalmology, Faculty of Medicine, Prince of Songkla University, Songkhla, \\ Thailand.
}

Received: March 27, 2021. Revised: August 3, 2021. Accepted: August 24, 2021. Published: August 26, 2021.

\begin{abstract}
Retinal fundus images are increasingly used by ophthalmologists both manually and without human intervention for detecting ocular diseases. Poor quality retinal images could lead to misdiagnosis or delayed treatment. Hence, a picture quality index was a crucial measure to ensure that the obtained images from acquisition system were suitable for reliable medical diagnosis.

In this paper, a no-reference retinal image quality assessment based on wavelet transform is presented. A multiresolution Daubechies (db2) wavelet at level 4 was employed to decompose an original image into detail, and approximation sub-bands for extracting the sharpness information. The sharpness quality index was calculated from the entropy of the sub-bands.

The proposed measure was validated by using images from the High-Resolution Fundus (HRF) dataset. The experimental results show that the proposed index performed more consistent with human visual perception and outperformed the Abdel-Hamid et al method.
\end{abstract}

Keywords-Image quality index, wavelet, entropy, retinal image.

\section{INTRODUCTION}

$\mathrm{R}$ etinal images are widely used to diagnose by ophthalmologists both with manual and without human intervention to identify and care for various eye diseases. Diagnosis accuracy is highly dependent on the quality of retinal images. The image can be degraded not only by capturing devices but also by aberrations caused by optical defects; especially, for the cloudy retinal image taken from cataract patient. Cataracts are the cause of cloudy vision where objects are obscured, blurred, and appear milky [1] [2]. This degraded image quality leads to incorrect diagnosis or delayed treatment. In this paper, a sharpness quality index is proposed for measuring and grading the color retinal images.

There are three categories of image quality measures: (1) full-reference approaches [3] [4], (2) reduced-reference approaches [5]; and (3) no-reference approaches [6] [7] [8] [9]. Full and reduced-reference approaches need the reference image for assessing the quality of the distorted image. Unfortunately, in retinal image acquisition, the reference images are not available. In this case, the no-reference approach suits best.

No-reference retinal image quality assessment (RIQA) algorithms compute a numeric quality index that is related to the visibility of the anatomical details in the retinal image. It has been conducted by many researchers. Crété-Roffet et al. [7] proposed a no-reference perceptual blur metric by measuring the relative difference of the luminance variation between the input image and the image burring version, which is achieved by convolving the input image with a low-pass filter. The more an image is blurred the less relative variation there is. Bahrami and Kot [8] proposed a no-reference image sharpness quality assessment by using a standard deviation of weighted maximum local variation (MLV) distribution to measure sharpness. However, the both quality assessment methods could not use to order the sharpness quality from the best to poor quality.

Recently, wavelet transform has been applied in most applications, such as steganography [10] and data compression [11] [12], especially in RIQA algorithms. Nirmala et al. [13] proposed a wavelet weighted blood vessel distortion to measure the green channel for quantification of the diagnostic information loss. The small and large blood vessels can be easily detected at levels 2 and 3 of the multiresolution wavelet filter process. However, this measure is not suitable for the cloudy retinal image of patients with a cataract.

Abdel-Hamid et al. [9] proposed a no-reference RIQA to assess the sharpness of the retinal images by employing the wavelet entropy [14]. The quality index $\left(\mathrm{Q}_{\mathrm{r}}\right)$ is calculated by dividing the entropy of the detail sub-bands, which is equivalent to the information of image sharpness, by the wavelet entropy of the approximation sub-bands, which is equivalent to the information of image background. Next, an image homogeneity parameter was considered to account for reduced image quality due to the nonvisible structures in the adequately illuminated regions of the retinal image. However, the border region between the region of interest (ROI) of retinal image and its 
black background leads to a problem through incorrect measurements.

This paper is motivated by the method of AbdelHamid et al. to overcome the drawbacks, it might be not correct order the image quality in HRF dataset. We study to design a quality index, which could sort the image quality of the dataset by the proposed index with corresponding to human vision system. The proposed index is developed to measure both the contrast and sharpness. Our image quality index is more consistent with human visual perception, and it could outperform the Abdel-Hamid et al method.

The paper proceeds as follows: the image database is introduced in Section II. The proposed method is described in Section III, our experimental results appear in Section IV, and discussion in Section V. Finally, the last paragraph introduces the main conclusions drawn.

\section{Materials}

A high-resolution fundus (HRF) image database [15] is applied to this work. The dataset images are captured with 18 image pairs of the same eye from 18 human subjects using a Canon CR-1 fundus camera with the Field of View (FOV) of $45^{\circ}$. For each pair, the quality of one image is good sharpness, whereas another is poor with slight blurs on the blood vessel and thus the image acquisition normally had to be repeated. Both bad and good images share approximately the same field of view. Therefore, the dataset images used for evaluation contain 18 bad and 18 good quality images.

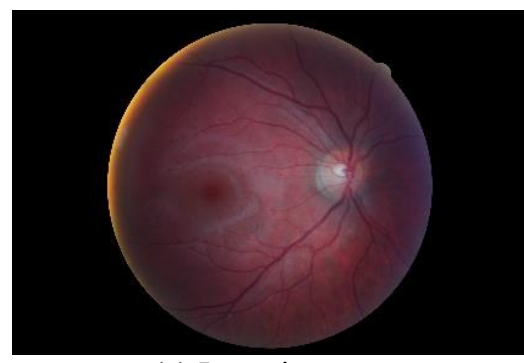

(a) Input image

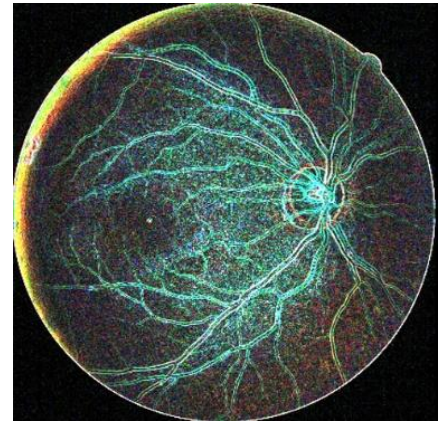

(c) Before remove border

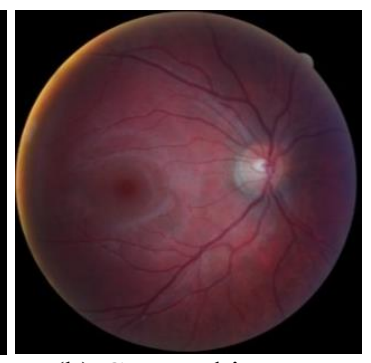

(b) Cropped image

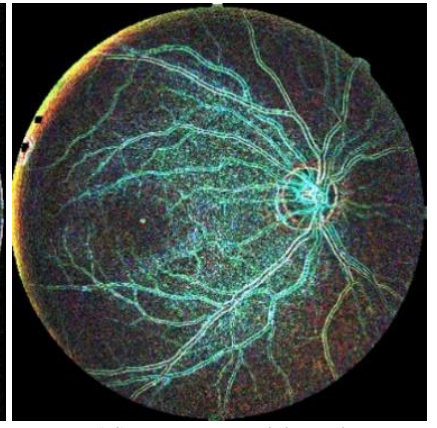

(d) Removed border.
Fig. 1 Preprocessing step consisting of cropping ROI, and removing the border regions between the ROI and background.

\section{The proposed method}

The high-quality retinal images are suitable for diagnosis. It easy to see the lesions that occur in the photographs. RIQA algorithm is therefore required in order to evaluate the image quality. Our RIQA method was designed to select the high-quality images. The proposed method consists of two steps. The first step supplies to preprocess the images consisting of cropping the ROI and removing the border between a background and the ROI as seen in Fig. 1. In Fig. 1, the input image has the size $3456 \times 5184$ pixels, after cropping the size of image is $3260 \times 3266$ pixels. From our study, the border between ROI and the background region appears after wavelet decomposition process as seen in Fig. 1(c). In our case, the border is noise, which is a cause to the method of AbdelHamid et al. unable to sort image quality of the dataset. Thus, in our method the border of all sub-band is removed as shown in Fig. 1(d).

In the second step, the quality index employed wavelet transform to objectively assess the sharpness of anatomical structures of the retinal images. Multiresolution Daubechies $(\mathrm{db} 2)$ wavelet filter is used to decompose the input image into horizontal $(H)$ and vertical $(V)$ detail sub-bands corresponding with the sharpness information of image foreground, and an approximation sub-band $(A)$ corresponding with the image background.

The proposed quality index, $Q_{\text {sharpness }}$, can be calculated by dividing the entropy of $H$ and $V$ by $A$ at level 4 as given:

$$
Q_{\text {sharpness }}=\frac{E(H)+E(V)}{E(A)}
$$

where $E(H), E(V)$, and $E(A)$ are the wavelet Shannon entropies of the horizontal, vertical and approximation sub-bands, respectively. The wavelet Shannon Entropy for each sub-band in Equation (1) is calculated using the following equation:

$$
E(C)=\left|\sum_{i=1}^{N} \log \left(C_{i}^{2}\right)\right|
$$

where $N$ is the number of coefficients in the wavelet sub-band, $C$ is the wavelet coefficient of Db2 at level 4 and $C_{i}$ is the wavelet coefficient having an index $i$ within the respective subbands.

To show that level 4 of wavelet multiresolution decomposition with $\mathrm{db} 2$ suits to measure the image sharpness, especially the cloudy retinal image, the image 1_bad.jpg from the HRF dataset is decomposed as shown in Fig. 2. This image is obscured and appears milky. Fig. 2 demonstrates the output results derived from each level of multi-scale Daubechies wavelet transform decomposition process. At level 1, the input image was first downsized by 2 and then decomposed with $\mathrm{db} 2$ resulting in an approximation sub-band $A_{1}$, and three detailed sub-bands, the horizontal $H_{1}$, vertical $V_{1}$, and diagonal $D_{1}$. By using the same process in level 1, all obtained sub-band outputs $C_{i}$, can be expressed as $C_{i}=\left\{A_{i}, H_{i}, V_{i}, D_{i}\right\}$ where $i$ is the scale level from 1 to 5 .

As mentioned in the introduction section, the study by Nirmala et al. [13] suggested that the small and large blood vessels can be easily seen at the second and third level. However, their study is not comprehensive in blurred regions that appear in the cloudy retinal images. 
By wavelet multiresolution decomposition with $\mathrm{db} 2$ in Fig. 2, the blurred regions in the partial image appeared clearly at $\mathrm{H}_{4}$ and $\mathrm{H}_{5}$. Those regions reduce the image sharpness and lead to the loss of the necessary anatomical structure details required for accurate interpretation. To determine the information quality of $\mathrm{H}_{4}(204 \times 204$ resolution $)$ and $\mathrm{H}_{5}$ with (102 $\times 102$ resolution), the $\mathrm{H}_{4}$ and $\mathrm{H}_{5}$ of Fig. 2 were magnified as shown in Fig. 3.

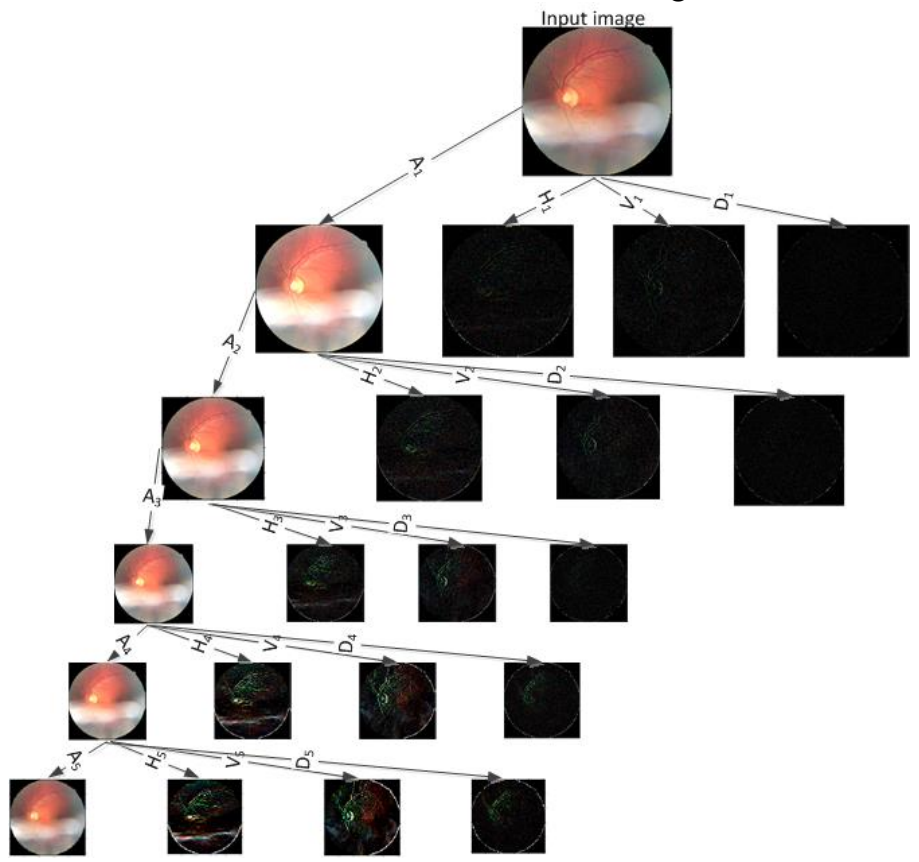

Fig. 2 Tree diagram of wavelet multiresolution decomposition with db2 from level 1 to 5 .

From Fig. 3, it is clearly seen that the blood vessel in $\mathrm{H}_{4}$ provides much more details and continuity than $\mathrm{H}_{5}$. This result shows that the coefficients of $\mathrm{H}_{4}$ sub-band has comparatively higher impact than $\mathrm{H}_{5}$ sub-band.

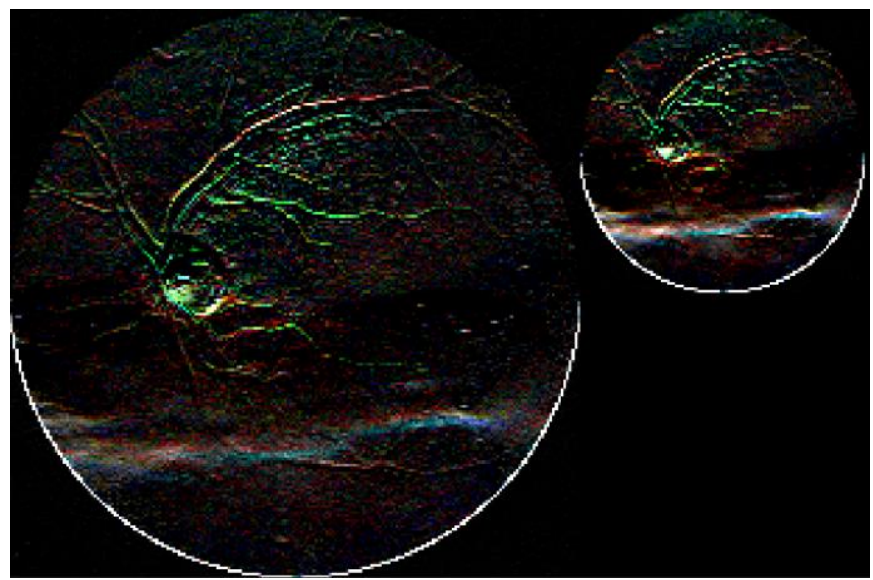

Fig. 3 Comparison of the detail information of $\mathrm{H}_{4}$ and $\mathrm{H}_{5}$.

Moreover, the horizontal detail sub-band of blood vessels is greater than the vertical, therefore, the information in $\mathrm{H}_{4}$ suits the most to measure the image sharpness. Fig. 4 shows a comparison of $\mathrm{H}_{4}$ between the blurred and sharp images. The $\mathrm{H}_{4}$ corresponding to Fig. 4 (a) and (b) are shown in (c) and (d), respectively.

Fig. 4 indicates that the $\mathrm{H}_{4}$ sub-band provided an obvious difference between the blurred and sharp image. This is the main reason why the proposed quality index calculated the entropy of approximation and detailed sub-bands at level 4.

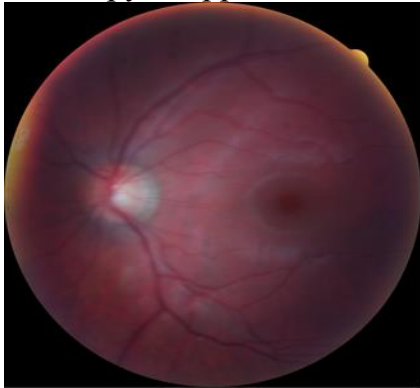

(a) 3 bad.jpg

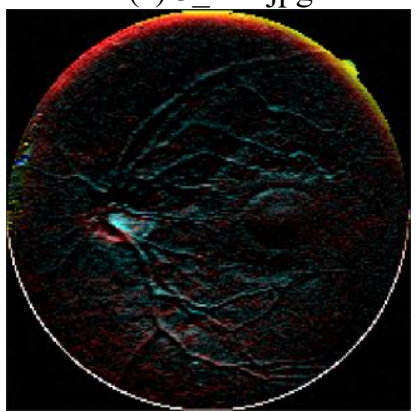

(c) $\mathrm{H}_{4}$ of image $(a)$

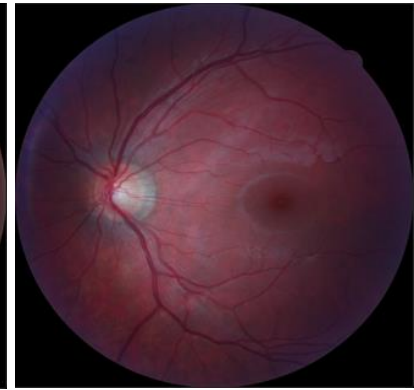

(b) 3 good.jpg

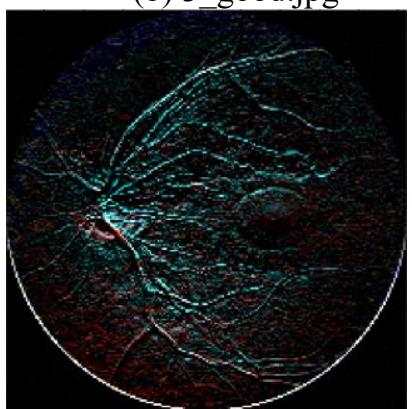

(d) $\mathrm{H}_{4}$ of image (b)
Fig. 4 Level 4 horizontal detail sub-band: $\mathrm{H}_{4}$ of blurry (a, c) and sharp $(b, d)$ retinal image from HRF.

\section{Experimental Results}

To evaluate the performance of our method, all images from the HRF dataset are used to compute the sharpness index 
with $Q_{\text {sharpness }}$ and Abdel-Hamid et al. method, $Q_{r}$. Table 1 shows $Q_{\text {sharpness }}$ and $Q_{r}$ values which are sorted by descending order. The first three columns comprise the order, filename, and sharpness index from the proposed method, respectively, while the last three columns are formulated by the
Abdel-Hamid et al. method. To compare the characteristics of the indexes, Fig. 5 depicts the graph of each image pair: bad and good from Table 1. Considering the line graphs of $Q_{\text {sharpness }}$, the quality index of good images remains constantly above all bad images.

Table 1 Comparison of $Q_{\text {sharpness }}$ and $Q_{r}$ value by using HRF dataset.

\begin{tabular}{|c|c|c|c|c|c|}
\hline \multicolumn{3}{|c|}{ The proposed method } & \multicolumn{3}{|c|}{ Abdel-Hamid et al. method [16] [17] } \\
\hline Order & Filename & $Q_{\text {sharpness }}$ & Order & Filename & $Q_{r}$ \\
\hline 1 & 12_good.JPG & 0.6588 & 1 & 2_good.JPG & 0.1864 \\
\hline 2 & 18_good.JPG & 0.6559 & 2 & 5_good.JPG & 0.0910 \\
\hline 3 & 9_good.JPG & 0.6559 & 3 & 16_good.JPG & 0.0850 \\
\hline 4 & 17_good.JPG & 0.6446 & 4 & 12_good.JPG & 0.0843 \\
\hline 5 & 8_good.JPG & 0.6446 & 5 & 17_good.JPG & 0.0706 \\
\hline 6 & 11_good.JPG & 0.6384 & 6 & 8_good.JPG' & 0.0706 \\
\hline 7 & 16_good.JPG & 0.6373 & 7 & 12_bad.JPG & 0.0700 \\
\hline 8 & 3_good.JPG & 0.6340 & 8 & 5_bad.JPG & 0.0690 \\
\hline 9 & 4_good.JPG & 0.6315 & 9 & 11_bad.JPG & 0.0687 \\
\hline 10 & 15_good.JPG & 0.6238 & 10 & 18_bad.JPG & 0.0683 \\
\hline 11 & 13_good.JPG & 0.6094 & 11 & 18_good.JPG & 0.0655 \\
\hline 12 & 5_good.JPG & 0.6074 & 12 & 9_good.JPG & 0.0655 \\
\hline 13 & 14_good.JPG & 0.6012 & 13 & 16_bad.JPG & 0.0626 \\
\hline 14 & 10_good.JPG & 0.5940 & 14 & 13_good.JPG & 0.0624 \\
\hline 15 & 7_good.JPG & 0.5921 & 15 & 11_good.JPG & 0.0622 \\
\hline 16 & 17_bad.JPG & 0.5693 & 16 & 14_good.JPG & 0.0605 \\
\hline 17 & 5_bad.JPG & 0.5682 & 17 & 9_bad.JPG & 0.0590 \\
\hline 18 & 7_bad.JPG & 0.5679 & 18 & 15_good.JPG & 0.0561 \\
\hline 19 & 12_bad.JPG & 0.5674 & 19 & 15_bad.JPG & 0.0521 \\
\hline 20 & 18_bad.JPG & 0.5513 & 20 & 10_good.JPG & 0.0495 \\
\hline 21 & 8_bad.JPG & 0.5338 & 21 & 14_bad.JPG & 0.0453 \\
\hline 22 & 4_bad.JPG & 0.5283 & 22 & 8_bad.JPG & 0.0452 \\
\hline 23 & 1_good.JPG & 0.5264 & 23 & 13_bad.JPG & 0.0443 \\
\hline 24 & 9_bad.JPG & 0.5139 & 24 & 2_bad.JPG & 0.0434 \\
\hline 25 & 11_bad.JPG & 0.5119 & 25 & 3_good.JPG & 0.0400 \\
\hline 26 & 14_bad.JPG & 0.4854 & 26 & 17_bad.JPG & 0.0378 \\
\hline 27 & 15_bad.JPG & 0.4804 & 27 & 1_good.JPG & 0.0323 \\
\hline 28 & 13_bad.JPG & 0.4718 & 28 & 4_bad.JPG & 0.0302 \\
\hline 29 & 16_bad.JPG & 0.4629 & 29 & 7_good.JPG & 0.0287 \\
\hline 30 & 3_bad.JPG & 0.4557 & 30 & 10_bad.JPG & 0.0270 \\
\hline 31 & 10_bad.JPG & 0.4407 & 31 & 6_good.JPG & 0.0259 \\
\hline 32 & 1_bad.JPG & 0.4261 & 32 & 6_bad.JPG & 0.0251 \\
\hline 33 & 2_good.JPG & 0.4221 & 33 & 7_bad.JPG & 0.0246 \\
\hline 34 & 6_good.JPG & 0.3953 & 34 & 1_bad.JPG & 0.0172 \\
\hline 35 & 6_bad.JPG & 0.3783 & 35 & 4_good.JPG & 0.0167 \\
\hline 36 & 2 bad.JPG & 0.3141 & 36 & 3 _bad.JPG & 0.0122 \\
\hline
\end{tabular}

When considering line graphs of $Q_{r}$, the graph lines of good and bad images have interfered. The results accumulated in Table 1, appear that the proposed method performs better than the Abdel-Hamid et al. method as most 
good images ranked higher and when compare with the same $\mathrm{x}$ images, there is no x_bad.jpg images with the $Q_{\text {sharpness }}$ values higher than $\mathrm{x}$ good.jpg images. In contrast, some $\mathrm{x}$ bad.jpg images highlighted in bold in Table 1, give higher
$Q_{r}$ values than x good.jpg such as the $Q_{r}$ values of 4_good.jpg in the $35^{\text {th }}$ order $(0.0167)$ is less than $Q_{r}$ values of 4_bad.jpg (0.0302).

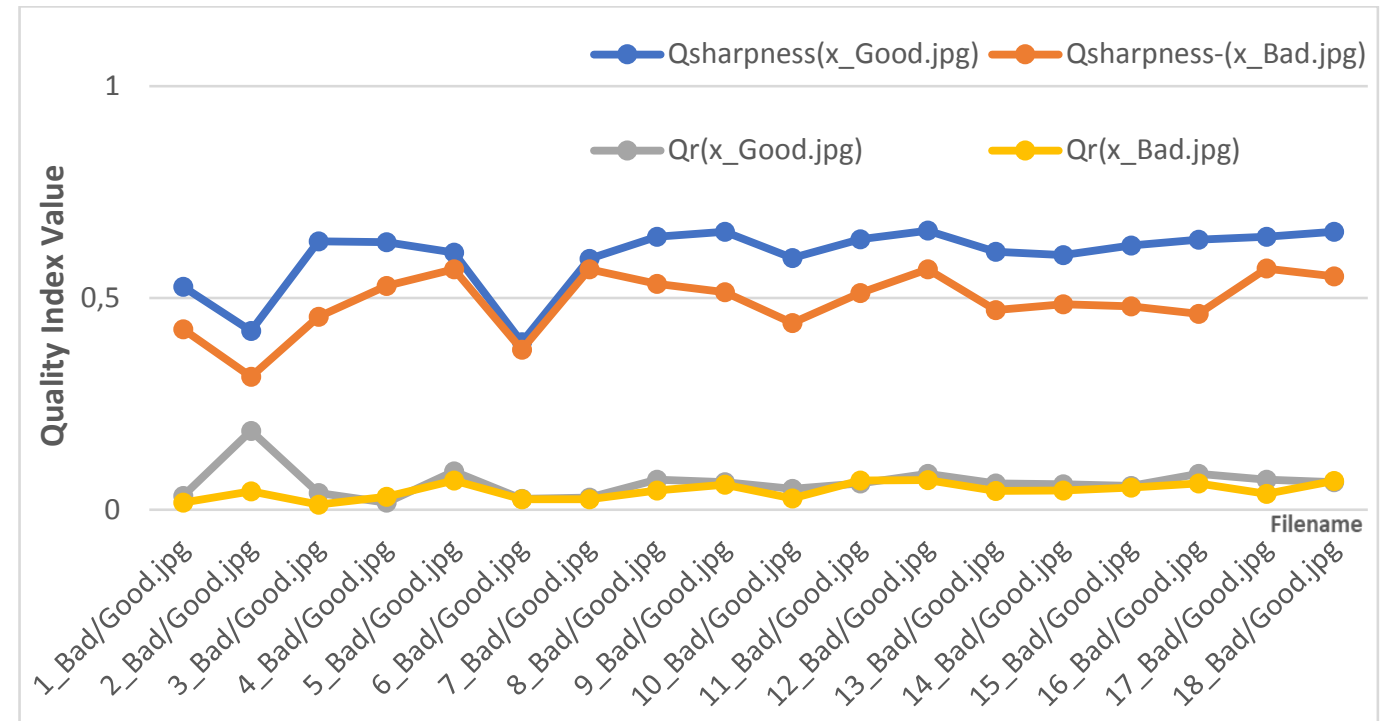

Fig. 5 The line graph of the quality index between the proposed and Abdel-Hamid et al. method for the same pair of good and bad images.

Fig. 6 shows the comparison of $Q_{\text {sharpness }}$ and $Q_{r}$ for the same pair of images, 4_good.jpg and 4_bad.jpg with $Q_{\text {sharpness }}=0.6315$ and 0.5283 while $Q_{r}=0.0167$ and 0.0302 , respectively. It can be seen that 4_good.jpg resulted in a greater $Q_{\text {sharpness }}$ value than the 4_bad.jpg image while the

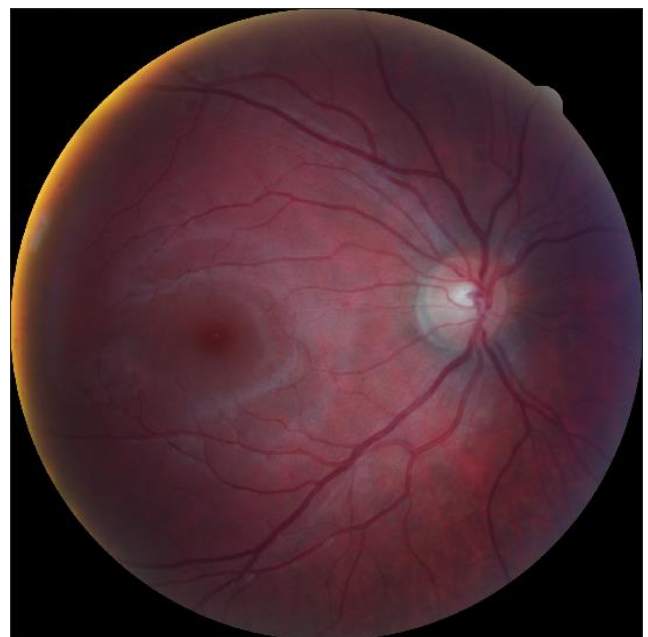

(a) 4_good.JPG

$Q_{\text {sharpness }}=0.6315, Q_{r}=0.0167$ 4_good.jpg image presented a smaller $Q_{r}$ value less than 4_bad.jpg. These results show that the proposed method provides more appropriate order than the Abdel-Hamid et al. method.

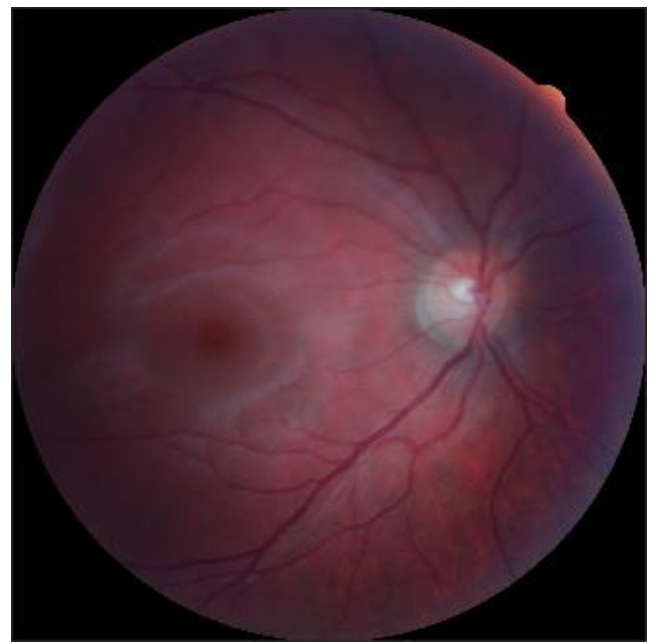

(b) 4_bad.JPG

$Q_{\text {sharpness }}=0.5283, Q_{r}=0.0302$

Fig. 6 Sharpness index comparison between $Q_{\text {sharpness }}$ and $Q_{r}$.

Fig. 7 highlights the images 12_good.JPG and 2_good.JPG from the HRF dataset with their image histograms where image 12_good.JPG reaches the maximum quality index of the proposed method $\left(Q_{\text {sharpness }}=0.6588\right)$ and image 2_good.JPG reaches the maximum quality index of the Abdel-
Hamid et al. method $\left(Q_{r}=0.1864\right)$. Although the image 2_good.JPG image provides great sharpness its contrast is quite low as seen in the image histogram. In addition, the image with low contrast should not be used for enhancement because the enhancement results in undesirable image tone and color 
balance. As a result, the image with low contrast should not have a high-quality index.
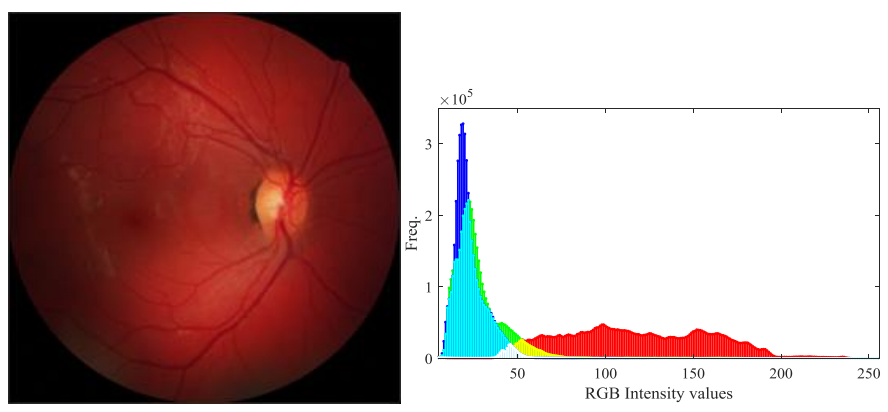

(a) 12_good.JPG

$Q_{\text {sharpness }}=0.6588$ [Order $\left.=1\right]$

$Q_{r}=0.0843$ [Order $\left.=4\right]$
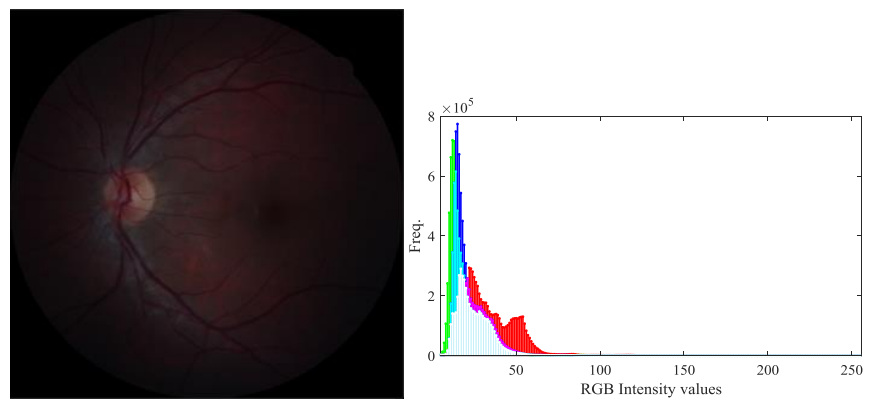

(b) 2 good.JPG

$Q_{\text {sharpness }}=0.4221$ [Order $\left.=33\right]$

$Q_{r}=0.1864[$ Order $=1]$

Fig. 7 Images with their image histograms that reached the maximum $Q_{\text {sharpness }}$ and $Q_{r}$.

Fig. 8 and Fig. 9 illustrated some photographs for visualize evaluation, which sorted from high to low quality indexes by using $Q_{r}$ and $Q_{\text {sharpness }}$, respectively. The photographs were selected by the same order at 1, 8, 15, 22, 29, and 36 as seen in Table 1. As seen in Fig. 8, the sequence of images sorted by the $Q_{r}$ value have several conflicts when comparing with the sequence that are sorted by $Q_{\text {sharpness }}$ in Fig. 9.

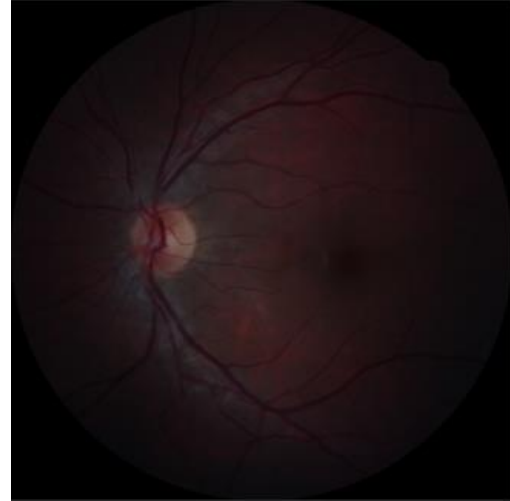

(a) 2_good.JPG, 0.1864

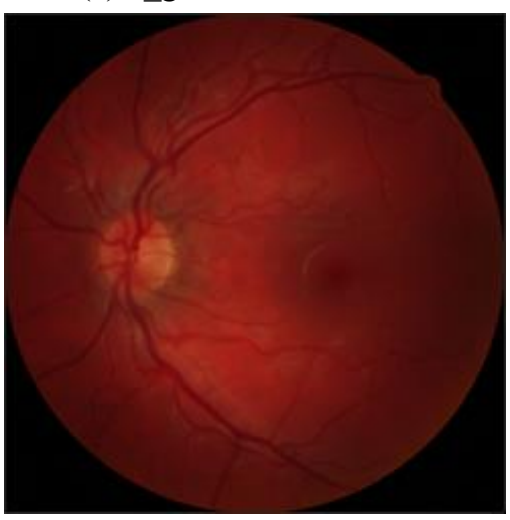

(d) 8_bad.JPG, 0.0452

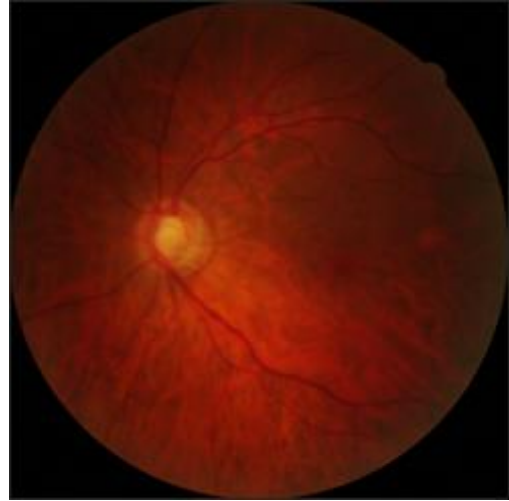

(b) 5 bad.JPG, 0.0690

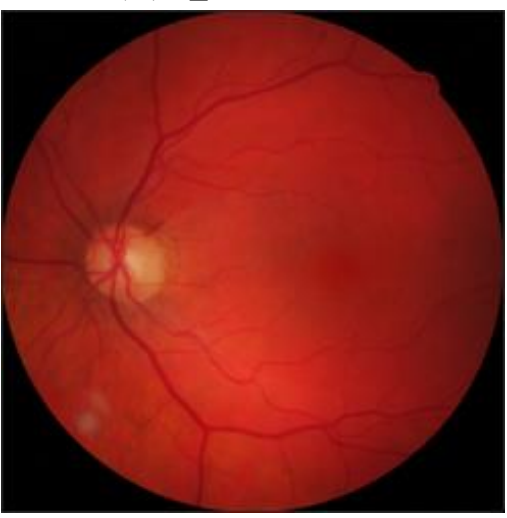

(e) 7_good.JPG, 0.0287

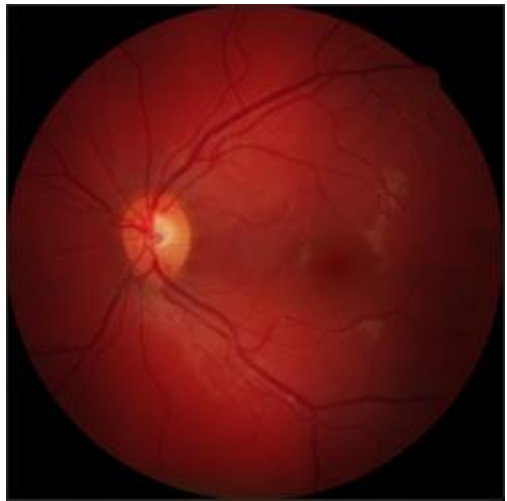

(c) 11 good.JPG, 0.0622

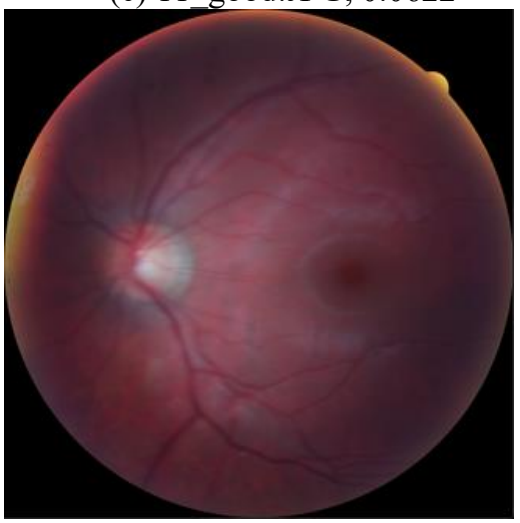

(f) 3_bad.JPG, 0.0122

Fig. 8 Example of image sequence sorted by $Q_{r}$. 


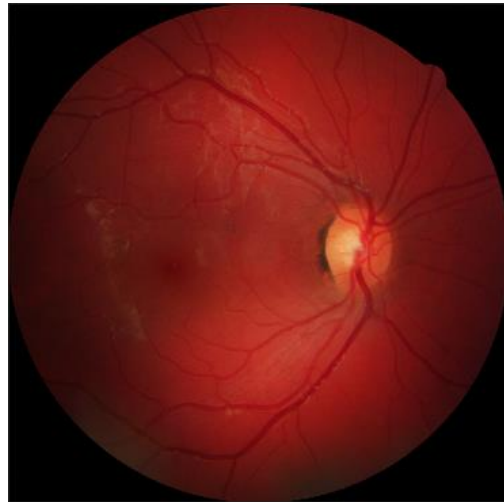

(a) 12 good.JPG, 0.6588

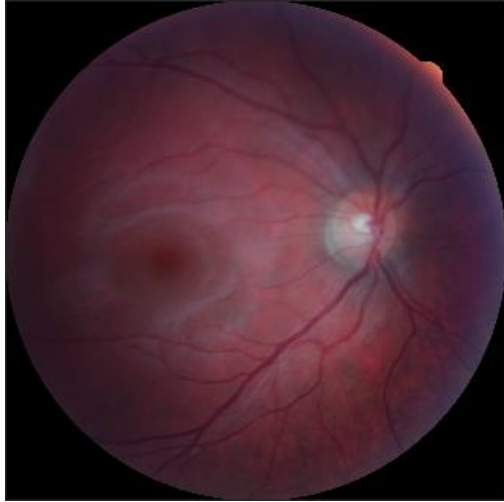

(d) 4 bad.JPG, 0.5283

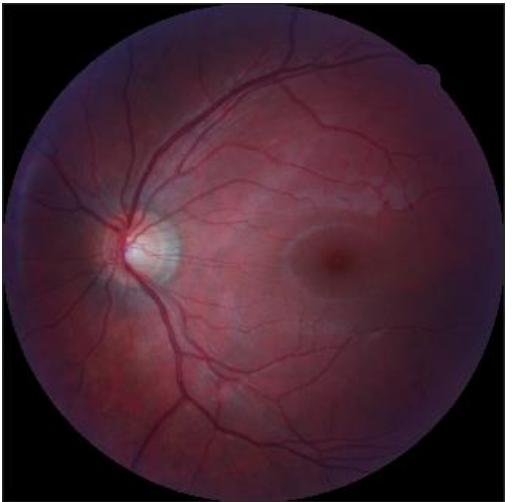

(b) 3 good.JPG, 0.6340

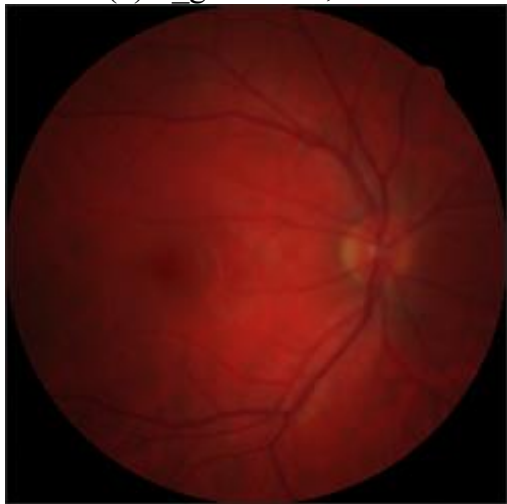

(e) 16 bad.JPG, 0.4629

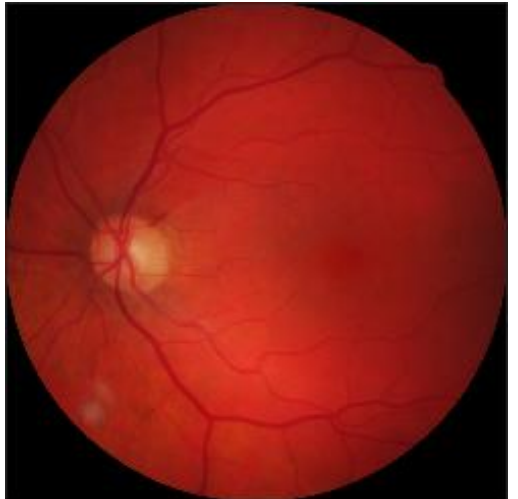

(c) 7 good.JPG, 0.5921

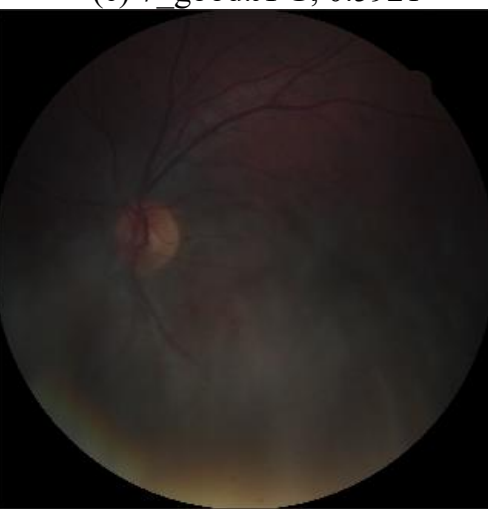

(f) 2_bad.JPG, 0.3141

Fig. 9 Example of image sequence sorted by $Q_{\text {sharpness }}$.

However, three good-quality images in Fig. 10 obtained a metric score lower than 0.5921. Although those three images are classified as good quality image [15] perceptionwise, they look unsatisfactory and show low correlation with human evaluations. Fig. 10 (a) appears an edge haze around the circular border which directly affect the image sharpness while many dark regions in Fig. 10 (b) and (c) are caused by nonuniform illumination.

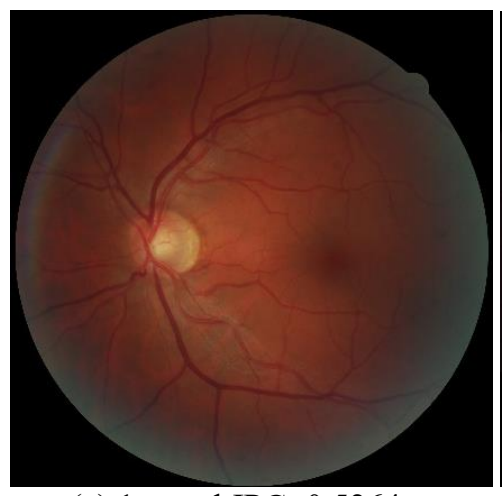

(a) 1 good.JPG, 0.5264

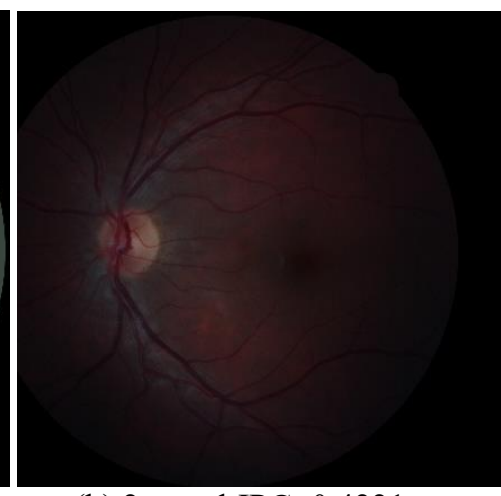

(b) 2_good.JPG, 0.4221

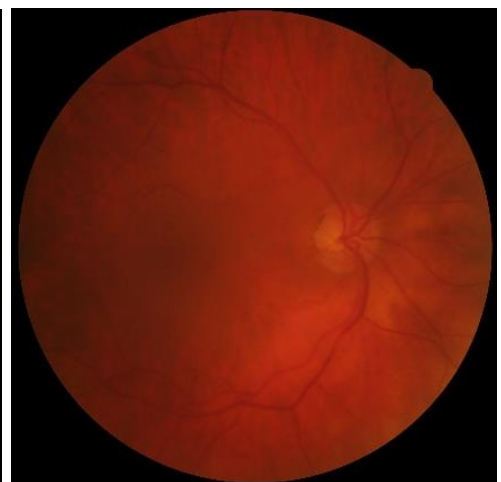

(c) $6 \_$good.JPG, 0.3953

Fig. 10 Example of good quality images with low $Q_{\text {sharpness }}$ value.

\section{Discussion}

The $\mathrm{Q}_{\text {sharpness }}$ index could be used in the quality evaluation procedure. Good quality images are accepted for further diagnosis by ophthalmologists, while bad quality images are rejected, and re-imaging is required. From Table1, images from order one to fifteen are good quality images. Hence, the minimum $\mathrm{Q}_{\text {sharpness }}$ value for a good quality image should be 0.6. As seen from Fig. 10, when $Q_{\text {sharpness }}$ less than 0.6 , those regions obscure the anatomical structures in the image. All artifacts in those three images decrease the quality of image [18] [19] and affect to the $Q_{\text {sharpness }}$ index.

\section{Conclusion}

A no-reference wavelet-based quality index was proposed to measure the sharpness of the color retinal images. The sharpness index is deduced from the ratio between the structural image in horizontal and vertical details and the blurriness in approximation sub-band, which is calculated by $\mathrm{db} 2$ at the $4^{\text {th }}$ 
level with the energy entropy. The index can measure the image quality as demonstrated in the results. Good and bad images could be compared as shown in Fig. 5; on the other hand, image quality could be arranged as reported in Table I. From the quality arrangement by our index, the retinal photographs can be used for diagnosis when the $Q_{\text {sharpness }}$ value is greater than or equal to 0.6 .

\section{References}

[1] A. M. Abdul-Rahman, T. Molteno and A. C. Molteno, "Fourier analysis of digital retinal images in estimation of cataract severity," Clinical and Experimental Ophthalmology, vol. 36, pp. 637-645, 2008.

[2] M. D. Abràmoff, M. K. Garvin and M. Sonka, "Retinal imaging and image analysis," IEEE Rev Biomed Eng., vol. 3, p. 169-208, 2010.

[3] Z. Wang and A. C. Bovik, "Mean squared error: love it or leave it? A new look at signal fidelity measures," Signal Processing Magazine, vol. 26, pp. 98-117, 2009.

[4] H.-S. Han, D.-O. Kim and R.-H. Park, "Structural information-based image quality assessment using LU factorization," IEEE Transactions on Consumer Electronics, vol. 55, pp. 165-171, 2009.

[5] Q. Li and Z. Wang, "General-purpose reduced-reference image quality assessment based on perceptually and statistically motivated image representation," in ICIP 2008, 2008.

[6] K. Panetta, C. Gao and S. Agaian, "No reference color image contrast and quality measures," IEEE

Transactions on Consumer Electronics, vol. 59, pp. 643651, 2013.

[7] F. Crete, T. Dolmiere, P. Ladret and M. Nicolas, "The Blur Effect: Perception and Estimation with a New NoReference Perceptual Blur Metric," in Proc. SPIE 6492, Human Vision and Electronic Imaging XII, 2007.

[8] K. Bahrami and A. C. Kot, "A Fast Approach for NoReference Image Sharpness Assessment Based on Maximum Local Variation," IEEE Signal Processing Letters, vol. 21, no. 6, pp. 751-755, 2014.

[9] L. Abdel-Hamid, A. El-Rafei and G. Michelson, "Noreference quality index for color retinal images," Computers in Biology and Medicine, vol. 90, pp. 68-75, 2017.

[10] S. Dutta and K. Saini, "Securing Data: A Study on Different Transform Domain Techniques," WSEAS TRANSACTIONS on SYSTEMS and CONTROL, vol. 16, pp. 110-120, 2021.

[11] Z. Gao and Y. F. Zheng, "Quality constrained compression using DWT-based image quality metric," IEEE Transactions on Circuits and Systems for Video Technology, vol. 18, no. 7, p. 910-922, 2008.

[12] K. P and J. M, "An Integrated Data Compression using Wavelet and Neural Networks for Power Quality Disturbances," WSEAS Transactions on Computer Research, vol. 7, pp. 9-22, 2019.
[13] S. Nirmala, S. Dandapat and P. Bora, "Wavelet weighted blood vessel distortion measure for retinal images," Biomedical Signal Processing and Control, vol. 4, no. 5, pp. 282-291, 2010.

[14] R. R. Coifman and M. V. Wickerhauser, "Entropy-based Algorithms for best basis selection," IEEE Trans. Inf. Theory, vol. 38, no. 2, p. 713-718, 1992.

[15] T. Köhler, A. Budai, M. Kraus, J. Odstrcilik, G. Michelson and J. Hornegger, "Automatic no-reference quality assessment for retinal fundus images using vessel segmentation," in Proceedings of the 26th IEEE International Symposium on Computer-Based Medical Systems, Porto, Portugal, 2013.

[16] L. Abdel-Hamid, A. El-Rafei and G. Michelson, "Noreference quality index for color retinal images," Computers in Biology and Medicine, vol. 8, pp. 68-75, 2017.

[17] L. Abdel-Hamid, A. El-Rafei, S. El-Ramly, G. Michelson and J. Hornegger, "Retinal image quality assessment based on image clarity and content," $J$. Biomed. Opt. SPIE, vol. 21, no. 9, pp. 1-17, 2016.

[18] R. C. Gonzalez and R. E. Woods, Digital Image Processing third edition, Pearson Prentice Hall, 2009.

[19] J. Lin, L. Yu, Q. Weng and X. Zheng, "Retinal Image Quality Assessment For Diabetic," Multimedia Tools and Applications, vol. 79, pp. 16137-16199, 2020.

\section{Author Contributions:}

Preecha Vonghirandecha organized the manuscript and executed the experiment.

Patama Bhurayanontachai was responsible for the experimental design and assessment.

Supaporn Kansomkeat was responsible for the algorithm testing.

Sathit Intajag proposed the algorithm, carried out the simulation, and executed the experiment.

\section{Creative Commons Attribution License 4.0 (Attribution 4.0 International, CC BY 4.0)}

This article is published under the terms of the Creative Commons Attribution License 4.0

https://creativecommons.org/licenses/by/4.0/deed.en_US 